\title{
Comparação entre dois modelos polinomiais para avaliar a tendência da linha de costa
}

\author{
Comparison between two polynomials models to evaluate shoreline tendency
}

\author{
Heithor Alexandre de Araújo Queiroz ${ }^{1}$ \\ Sydney de Oliveira Dias² \\ Rodrigo Mikosz Gonçalves ${ }^{1}$ \\ Pedro de Souza Pereira ${ }^{3}$
}

\begin{abstract}
${ }^{1}$ Departamento de Engenharia Cartográfica, Programa de Pós-Graduação em Ciências Geodésicas e Tecnologias da Geoinformação, Universidade Federal de Pernambuco - UFPE. Avenida Professor Morais Rego, 1235 - Cidade Universitária. Cep 50670-901. Recife, PE, Brasil.

${ }^{2}$ Departamento de Design, Infraestrutura e Meio Ambiente, Instituto Federal de Educação, Ciência e Tecnologia da Paraíba - IFPB. Avenida $1^{\circ}$ de Maio, 720 - Jaguaribe. Cep 58015-430. João Pessoa, PB, Brasil.

${ }^{3}$ Departamento de Oceanografia, Instituto Nacional de Ciência e Tecnologia em Ambientes Marinhos Tropicais INCT AmbTropic, Universidade Federal de Pernambuco. Avenida Professor Morais Rego, 1235 - Cidade Universitária. Cep 50670-901. Recife, PE, Brasil.

heithorqueiroz@gmail.com, sydney.dias@ifpb.edu.br,rodrigo.mikosz@ufpe.br, pedro.pereira@pq.cnpq.br
\end{abstract}

Recebido em 04/02/2016 - Aceito em 09/12/2016

Received on 04/02/2016 - Accepted on 09/12/2016

\begin{abstract}
RESUMO - A linha de costa é uma feição dinâmica e o conhecimento do seu comportamento posicional no presente, passado e possíveis tendências torna-se fundamental para o planejamento e gestão costeira integrada. O objetivo desta contribuição científica foi avaliar a tendência da linha de costa aplicando o Método dos Mínimos Quadrados em uma série temporal comparando dois modelos matemáticos: o polinomial linear e o quadrático. A área de estudo contempla a Praia do Seixas, Paraíba, através de observações provenientes de ortofotos, imagens de satélite e levantamentos GNSS, totalizando desta forma 8 observações temporais para um intervalo de 17 anos. Para avaliar os modelos matemáticos escolhidos foram obtidas as tendências para o ano de 2015. Como resultado de tendência dos modelos têm-se a comparação com a linha de costa de 2015 apresentando, para o setor norte, discrepância de 3,6m e 8,9m e para o setor sul de 3,0m e 11,4m considerando o modelo linear e o quadrático, respectivamente. Quando comparado a tendência a longo prazo, neste caso considerando um horizonte de 40 anos, foi possível verificar diferenças na ordem de $200 \mathrm{~m}$ entre os modelos matemáticos testados. As discrepâncias entre as predições calculadas pelo modelo linear foram menores que as alcançadas pelo modelo quadrático, nos dois setores. Por outro lado foram obtidos valores de $\mathrm{R}^{2}$ mais próximos de um (1) para o modelo polinomial quadrático. Os resultados indicam que é necessário ter cautela na escolha de um modelo matemático para o caso do comportamento de tendência da linha de costa.
\end{abstract}

Palavras-chave: linha de costa, tendência, modelo matemático polinomial.

ABSTRACT - Shoreline is a dynamic continental feature and it is important to know its behavior in the present, past and future. The aim of this paper is to evaluate the shoreline trend applying the method of least squares in a temporal series of data comparing the polynomials models of first order and second order. The chosen study area was Seixas Beach, Paraiba, Brazil. The data available cover 17 years and it was obtained using orthophotos, satellite images and GNSS survey. To evaluate these mathematic models it was calculated the shoreline trend to 2015. The results obtained through the comparison between 2015 shoreline and the trends obtained using the two methods showed to the linear and quadratic models respectively $3.6 \mathrm{~m}$ and $8.9 \mathrm{~m}$ to north zone and $3.0 \mathrm{~m}$ and $11.4 \mathrm{~m}$ to south zone. Then it was generated the long term trend covering 40 years and it showed approximately $200 \mathrm{~m}$ of difference between the models. The differences between the trends were lower to first order polynomial model in both sectors. On the other hand, the second order model showed $\mathrm{R}^{2}$ values nearest 1 . It is important to notice the results indicate that is necessary to be careful about the choice of the mathematical models to obtain the shoreline trends.

Keywords: shoreline, trend, polynomial mathematic model.

\section{INTRODUÇÃO}

A linha de costa pode ser definida como a interface física entre terra e água (DOLAN et al., 1980). Segundo
$\mathrm{Li}$ et al. (2002), o International Geographic Data Committe (IGDC) reconheceu a linha de costa como sendo uma das 27 feições terrestres mais importantes. 
A linha de costa está sujeita a alterações contínuas, e este comportamento instável pode não ter um padrão na escala temporal, e assim apresentar variações entre diferentes épocas, sejam dias, décadas ou séculos (WHITE, 2007).

Esta dinamicidade espaço-temporal pode estar relacionada a diversos fatores, tanto naturais, a exemplo das alterações no nível do mar, condições de maré, mudanças climáticas, ocorrências de tempestades, ação dos ventos, movimentação das ondas, deslocamento de sedimentos ocasionados por rios e pelo oceano, processos geomorfológicos de erosão e progradação costeira, quanto antrópicos, como implantação de obras de engenharia na costa, remoção intencional de sedimentos (retirada para uso em obras) ou não intencional (ocasionadas pelo uso recreacional) (CURRIN et al., 2015; DAN et al., 2012; MANCA et al., 2013; UMAR et al., 2013).

A configuração do comportamento da linha de costa é, portanto, um reflexo das mudanças da natureza a nível global, unidas aos processos de mudanças no ambiente costeiro e às atividades humanas. Estes diversos fatores, que influenciam na dinâmica da sua posição, justificam a afirmação de que a definição da linha de costa é simples, porém a sua detecção é laboriosa (BOAK; TURNER, 2005; DAN et al., 2012;).

A detecção da linha de costa depende da escolha do indicador da posição e do reconhecimento deste indicador nas fontes de dados disponíveis. Um indicador é uma feição utilizada para a representação da posição da linha de costa. São exemplos: a linha de maré alta (high water line - HWL) - que se refere à marca deixada pela água no espraio das ondas, e exigem uma interpretação visual humana para a extração da feição -, o nível médio da maré alta (Mean High Water Line - MHWL) - que usa como referência a interseção entre o perfil de praia e o datum altimétrico adotado (altura de maré). (BOAK; TURNER, 2005).

São diversas as técnicas e as fontes de dados utilizadas para extração de informações da linha de costa. Estas provem de dados históricos, que são utilizados para a obtenção das taxas de variação da linha de costa ao longo do tempo. São exemplos os produtos do sensoriamento remoto, como, fotografias aéreas e imagens de satélites, bem como os levantamentos obtidos pelo uso do GNSS, entre outras fontes (BOAK; TURNER, 2005)

Existem vários modelos matemáticos usados para obter as taxas de variações da posição da linha de costa como, por exemplo, método dos pontos finais (End Point Rate - EPR), método da média dos pontos (Average of Rates - AOR), Jackknifing (JK) entre outros, que podem ser vistos com detalhes em Genz et al. (2007). Já para verificar a tendência a curto prazo é comum encontrar na literatura os modelos de regressão linear, estimativa robusta, ou redes neurais artificiais, como exemplo cita-se o trabalho de Gonçalves et al. (2010) a respeito de redes neurais artificiais e Gonçalves (2010) comparando estes três modelos para um estudo de caso na praia de Matinhos no Paraná.
De certa forma o modelo comumente utilizado para estimativa da tendência da linha de costa é a regressão linear, porém destaca-se que este modelo é indicado quando os dados atendem a distribuição normal, como também quando se é considerada apenas a tendência linear de erosão e são relevados outros fatores como ocorrências de tempestades (APPEANING ADDO et al., 2008; DOUGLAS; CROWELL, 2012; GONÇALVES et al., 2012).

Appeaning Addo et al. (2008) salientam que as taxas de variação são afetadas pelas ondas e mudanças de maré a curto prazo, pela alteração no nível do mar a longo prazo, como também pelo transporte de sedimentos ao longo da costa, por conseguinte acrescenta-se uma imprecisão aos valores obtidos.

Essas contínuas variações no nível do mar fazem com que as taxas calculadas para determinado intervalo de tempo não retratem com fidelidade o comportamento futuro da linha de costa. Sendo, portanto, necessário um maior cuidado na análise do ajuste do modelo matemático que está sendo usado para descrever o comportamento da linha de costa.

De acordo com Crowell et al. (1993), para a avaliação da tendência do comportamento da linha de costa o ideal é que os dados utilizados no cálculo das taxas de variação histórica para determinada área compreendam um intervalo de, no mínimo, 60 a 80 anos, e que a acurácia dos dados seja analisada (APPEANING ADDO et al., 2008). Porém na prática nem sempre é possível obter uma série longa de dados históricos.

Considerando essa temática, este trabalho teve como objetivo avaliar as tendências da posição da linha de costa obtidas por dois modelos polinomiais, o linear e o quadrático, utilizando o Método dos Mínimos Quadrados (MMQ) aplicado à área de estudo, a Praia do Seixas na Paraíba. A seguir apresentam-se a localização da área de estudo no item 2, os materiais e métodos (item 3) bem como os resultados e discussão no item 4 e por fim as conclusões no item 5 .

\section{2. ÁREA DE ESTUDO}

A área escolhida para o estudo é mostrada na Fig. 1, e refere-se à Praia do Seixas do município de João Pessoa, $\mathrm{PB}$, pertencente à Região Nordeste do Brasil.

Com uma extensão de aproximadamente $2,8 \mathrm{~km}$, é caracterizada pela presença de escarpas, e predomínio de areias finas, além de apresentar uma tendência erosiva e alta vulnerabilidade à erosão costeira (eg. Reis, 2008).

Além disso, a Praia do Seixas tem um elevado potencial turístico, pois compreende a Ponta do Seixas, que é o ponto peninsular mais oriental das Américas e algumas obras importantes como o Farol do Cabo Branco e a Estação Cabo Branco (obra assinada pelo arquiteto Oscar Niemeyer). 


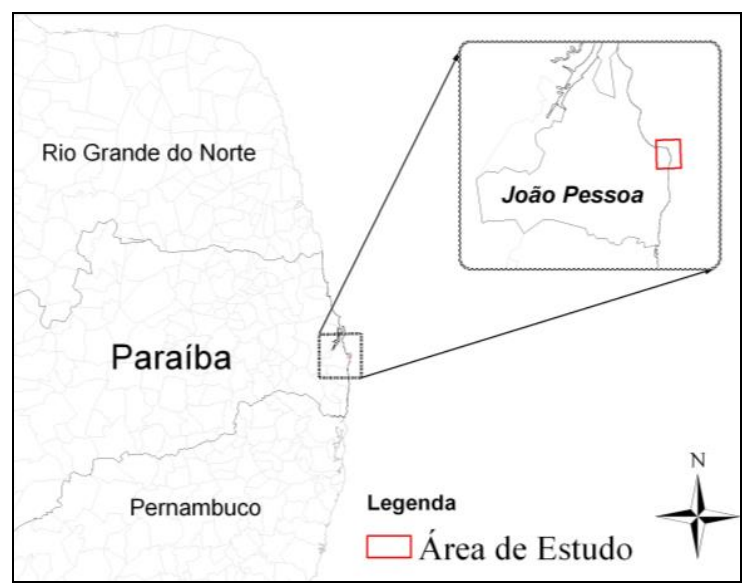

Figura 1 - Área de Estudo: Praia do Seixas, João Pessoa, PB. Fonte: $\mathrm{O}$ autor.

\section{MATERIAIS E MÉTODOS}

As observações posicionais da linha de costa, utilizadas neste trabalho, referem-se aos anos 1998, 2005, 2006, 2008, 2012, 2013, 2014 e 2015. As edições, processamentos, bem como levantamentos, para a obtenção dessas observações foram realizadas pela equipe do Laboratório de Geoprocessamento (LabGeo) do Instituto Federal da Paraíba (IFPB).

Parte dos dados foi obtida através da vetorização de imagens de satélites e de ortofotos, de resolução espacial submétrica. Foi considerada como indicador da linha de costa a marca d'água deixada pelo espraio das ondas, ou seja, o encontro entre a parte seca da praia e a molhada identificada nas imagens e caracterizado desta forma o alcance máximo da maré para determinado instante na obtenção das imagens. $\mathrm{O}$ uso de um indicador para representar a linha de costa é muito comum em estudos costeiros e, uma vez escolhida esta feição, este valor é considerado como "verdadeiro". Mais informações de como extrair e escolher indicadores da linha de costa podem ser encontradas em Boak e Turner (2005).

Anteriormente ao processo de vetorização foi realizado o procedimento para o registro das imagens. Para isto foram efetuados levantamentos de campo para obtenção dos pontos de controle, por meio de levantamentos GNSS e método de posicionamento relativo estático.

Para o registro das imagens optou-se em utilizar o modelo matemático de $1^{\circ}$ ordem, uma transformação afim considerando seis parâmetros. Os pontos de controle, levantados em campo, foram utilizados para a correção da imagem mais atual (Quickbird, 2008) e as demais imagens foram reamostradas tomando-se esta como referência.

As observações de 1998 e 2006 são oriundas de digitalizações manuais feitas através de interpretação de fotografias aéreas convencionais no formato digital. Este mesmo procedimento foi utilizado para a extração da linha de costa para os anos 2005 e 2008 sobre as duas imagens de alta resolução do sensor CCD do satélite QuickBird.

Os dados referentes aos anos 2012, 2013, 2014 e 2015 foram obtidos por meio de levantamentos geodésicos, através do uso do GNSS, utilizando o método RTK (Real Time Kinematic).

Os levantamentos ocorreram nos períodos em que a altura da maré registrava leitura próxima a 2,8 metros, valor este considerado como o máximo alcance das marés para a área de estudo, conforme a Diretoria de Hidrografia e Navegação (DHN). Portanto foi adotado como indicador da posição da linha de costa a HWL, ou seja, a altura máxima de alcance da água.

\subsection{Pré-análise da série temporal e organização das observações}

Para o cálculo da variação entre as linhas de costa, foi utilizado uma linha de base (referência) de acordo com o método descrito por Leatherman e Clow (1983). Neste método recomenda-se que a linha de referência seja estabelecida de forma adjacente ao conjunto que compõe a série temporal de linhas de costa, com o cuidado de preservar a mesma orientação das demais linhas. A partir da linha de base são traçados de maneira perpendicular os transectos, os quais são usados para calcular o deslocamento entre as linhas de costa.

De acordo com as características da área estudada, optou-se em gerar transectos com equidistância de 50 metros, totalizando assim 51 transectos. Cada um destes cruza em algum ponto as linhas de costa de todas as amostras temporais, entre 1998 e 2014. A partir daí, efetuou-se o cálculo das distâncias entre a linha de base e a linha de costa para cada ano.

A partir destas observações foi possível identificar os padrões de comportamento da linha de costa no decorrer dos anos em cada transecto. Posteriormente a área de estudo foi dividida em dois setores, norte e sul, cerca de 30 e $70 \%$ da extensão total, respectivamente (Fig. 2). 

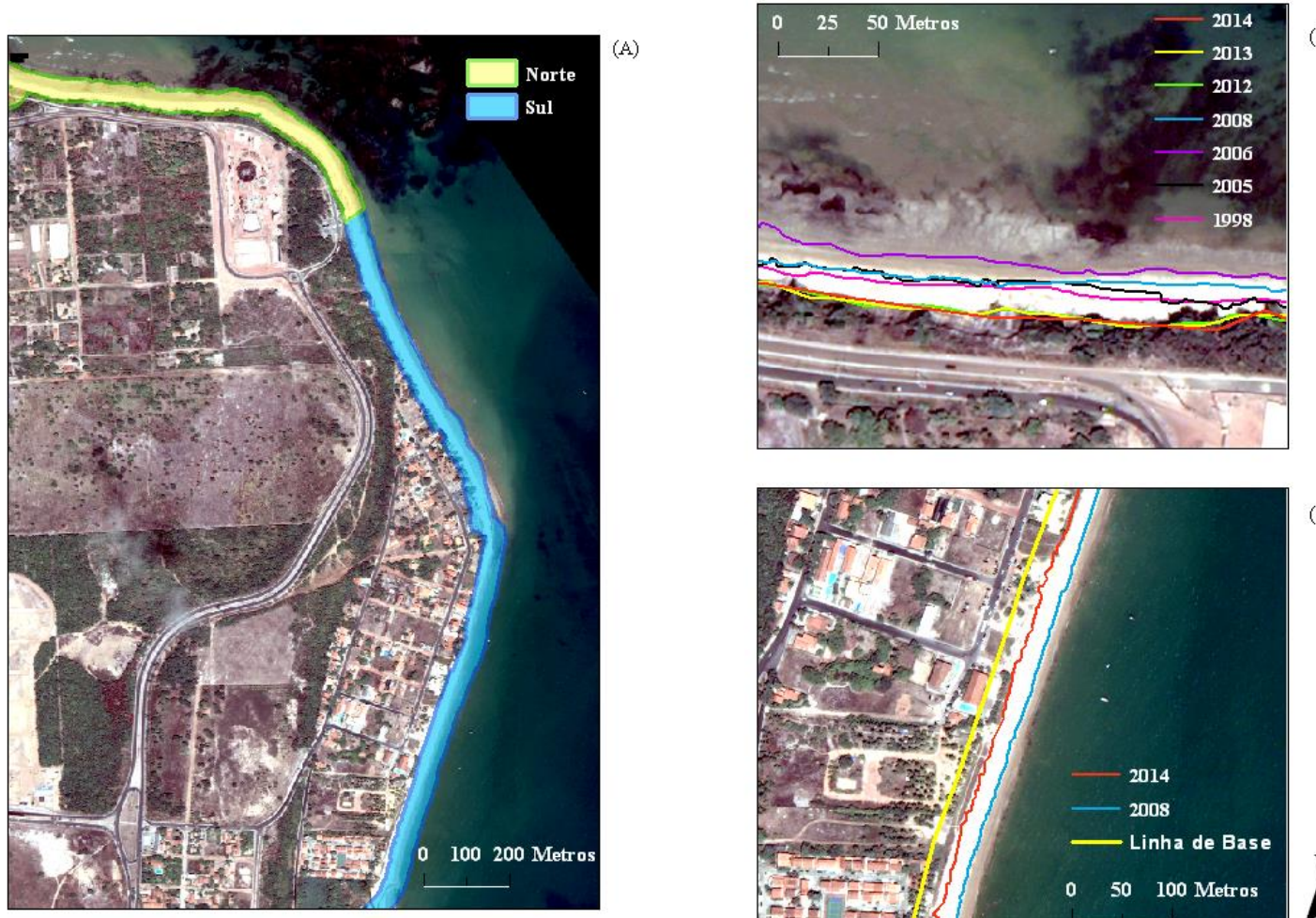

(B)

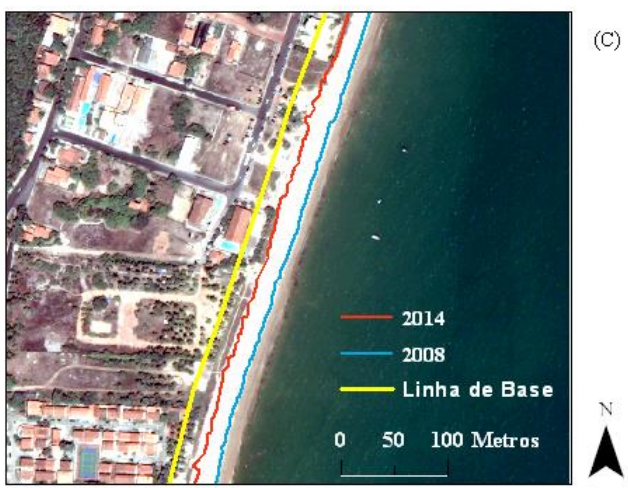

Figura 2 - (A) Divisão da área de estudo em setores (Norte e Sul); (B) Trecho do setor norte com linhas de costa entre 1998 e 2014 sobrepostas a uma imagem de alta resolução espacial (QuickBird) do ano de 2008. (C) Trecho do setor sul com a linha de costa de 2008, vetorizada sobre a imagem a qual está sobreposta e linha de costa de 2014 (oriunda de mapeamento GNSS/RTK - in situ) e também a linha de base (referência).

O setor norte compreende um trecho com características mais uniformes na variação das distâncias ao longo dos transectos no decorrer dos anos, mostrado na Fig. 3. Já o setor sul, pelo contrário, não apresenta esta uniformidade, como pode ser visto na Fig. 4. Optou-se neste caso em não subdividir o setor Sul justamente para verificar o comportamento dos modelos em casos onde os padrões são diferentes.

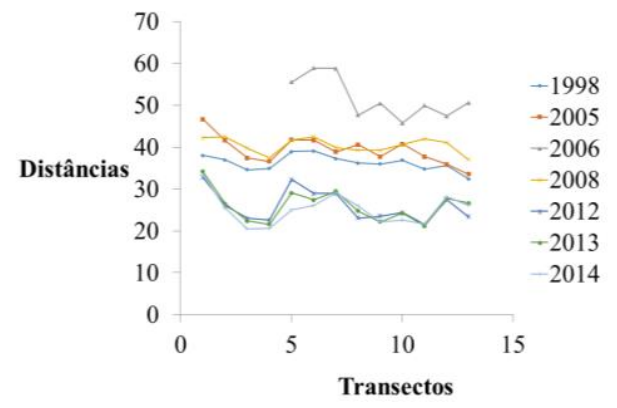

Figura 3 - Setor norte: distância entre linha de base e linha de costa versus transectos (1 a 13).

Fonte: O autor.

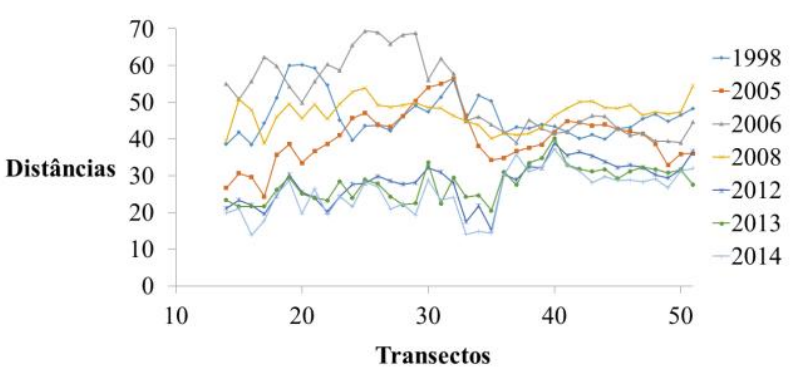

Figura 4 - Setor sul: distância entre linha de base e linha de costa versus transectos (14 a 51).

Fonte: $\mathrm{O}$ autor.

É possível perceber uma maior uniformidade na variação das distâncias no setor norte (Fig. 3), enquanto no setor sul (Fig. 4) as distâncias para cada ano variam muito no decorrer dos transectos dispostos ao longo da área de estudo. Cabe salientar que para o ano de 2006 há um vazio de informações entre o transecto de número 1 até o de número 4 , mas isso não interfere no experimento proposto.

As maiores acresções ocorreram em 2006, em ambos os setores, enquanto que as maiores erosões ocorreram nos anos mais atuais de 2012, 2013 e 2014, em ambos os setores. 
De posse das distâncias obtidas a partir dos transectos entre a linha de base e a linha de costa para cada ano, foi calculado a média aritmética, em metros, representando um valor de deslocamento médio para cada ano (de 1998 a 2014), conforme o setor analisado. Estes valores obtidos são apresentados na Tab. 1, os mesmos foram considerados como parâmetros de entrada para a regressão dos modelos linear e quadrático conforme a matriz das observações $(L a)$ no modelo de ajustamento paramétrico que será descrito no item 3.2.

Tabela 1 - Média amostral das distâncias, em metros, contadas sobre os transectos, entre a linha de base e a linha de costa de cada ano.

\begin{tabular}{cccc}
\hline \multicolumn{2}{c}{ SETOR NORTE } & \multicolumn{2}{c}{ SETOR SUL } \\
\hline Ano & Média (m) & Ano & Média (m) \\
\hline 1998 & 36,26 & 1998 & 46,26 \\
2005 & 39,28 & 2005 & 40,34 \\
2006 & 51,67 & 2006 & 51,56 \\
2008 & 40,38 & 2008 & 47,11 \\
2012 & 25,97 & 2012 & 28,43 \\
2013 & 25,93 & 2013 & 27,86 \\
2014 & 25,16 & 2014 & 25,51 \\
\hline
\end{tabular}

Também foram obtidas as distâncias entre a linha de base e a linha de costa para o ano de 2015, servindo como "valor verdadeiro" para a comparação com o valor obtido pelos modelos matemáticos. Foi calculada a média amostral destas distâncias, bem como os desvios-padrão (DP), em metros, para os setores norte e sul. Estes valores estão expressos na Tab. 2.

Tabela 2 - Média amostral e desvio-padrão das distâncias, em metros, entre a linha de base e a linha de costa para o ano de 2015 , contadas sobre os transectos, tidas como o "dado real" para o posterior experimento.

\begin{tabular}{cccccc}
\hline \multicolumn{3}{c}{ SETOR NORTE } & \multicolumn{3}{c}{ SETOR SUL } \\
\hline Ano & Média & DP & Ano & Média & DP \\
\hline 2015 & 23,63 & $+-3,92$ & 2015 & 24,77 & $+-6,55$ \\
\hline
\end{tabular}

\subsection{Método dos Mínimos Quadrados - Modelo Paramétrico}

Para se obter os parâmetros ajustados dos modelos polinomiais testados foi usado o modelo paramétrico, descrito por (GEMAEL, 1994):

$$
L a=A X a
$$

Já que $\mathrm{La}=\mathrm{Lb}+\mathrm{V}$, portanto a Eq. 1 pode ser reescrita da seguinte maneira:

$$
L b=A X a-V
$$

Em que:

La - vetor (n x 1) das observações ajustadas;
$\mathrm{Lb}$ - vetor (n x 1) das observações;

Xa - vetor (u x 1) dos parâmetros ajustados;

A - matriz ( $\mathrm{n} \times \mathrm{u})$ dos coeficientes;

$\mathrm{V}$ - vetor (n x 1) dos resíduos.

A partir da Eq. 2, obtém-se que:

$V=A X a-L b$

O vetor dos parâmetros ajustados (Xa) é obtido aplicando o critério dos mínimos quadrados :

$$
V^{T} P V=\text { mínimo }
$$

$$
X a=\left(A^{T} P A\right)^{-1} A^{T} P L b
$$

$\mathrm{ou}$

$$
X a=N^{-1} U
$$

onde:

$\mathrm{N}$ - matrizes ( $\mathrm{u} \times \mathrm{u}$ ) dos coeficientes das equações normais;

$\mathrm{U}$ - vetores ( $\mathrm{u} \times 1$ ) dos termos independentes;

$\mathrm{P}$ - matriz peso ( $\mathrm{n} \times \mathrm{n})$ das observações.

A partir destas informações (Tab. 1), foi aplicado o MMQ para se obter os parâmetros dos modelos matemáticos polinomiais de $1^{\mathrm{a}}$ e $2^{\mathrm{a}}$ ordem, tanto para o setor norte, quanto para o sul. Também foi calculado o coeficiente de determinação $\mathrm{R}^{2}$, que indica a qualidade do ajuste do modelo, onde a proximidade em relação a um (1) indica o melhor ajuste obtido pelo modelo matemático.

$\mathrm{Na}$ sequência foram calculados os valores de tendência da posição da linha de costa para o ano de 2015, usando os parâmetros encontrados. Estes valores foram comparados com as distâncias para o ano de 2015 mostradas anteriormente na Tab. 2.

Por conseguinte, foram calculadas as tendências para os anos de 2020, 2025, 2030, 2035, 2040, para os dois setores, norte e sul e respectivos modelos matemáticos.

\section{RESULTADOS E DISCUSSÃO}

Os valores dos parâmetros obtidos através do MMQ para os modelos polinomiais linear (primeira ordem) e quadrático (segunda ordem), bem como o coeficiente de determinação $\left(\mathrm{R}^{2}\right)$ de cada modelo para cada conjunto de amostra divididas entre os setores norte e sul, são mostrados nas Tab. 3 (norte) e Tab. 4 (sul). 
Tabela 3 - Valores dos parâmetros obtidos através do MMQ e o coeficiente de determinação $\left(\mathrm{R}^{2}\right)$ de cada modelo para o setor norte.

\begin{tabular}{ccc}
\hline \multicolumn{3}{c}{ SETOR NORTE } \\
\hline Modelo & Parâmetros & $\mathrm{R}^{2}$ \\
\hline Linear (1 $1^{\mathrm{a}}$ ordem) & $a=-1,049$ & 0,3559 \\
& $b=2141$ & \\
Quadrático (2 ${ }^{\mathrm{a}}$ ordem) & $a=-0,227836285$ & 0,8005 \\
& $b=913,1938890219$ & \\
\hline & $c=-914986,508911132$ \\
\hline
\end{tabular}

Tabela 4 - Valores dos parâmetros obtidos através do MMQ e o coeficiente de determinação $\left(\mathrm{R}^{2}\right)$ de cada modelo para o setor sul.

\begin{tabular}{ccc}
\hline \multicolumn{3}{c}{ SETOR SUL } \\
\hline Modelo & Parâmetros & $\mathrm{R}^{2}$ \\
\hline Linear (1 ${ }^{\mathrm{a}}$ ordem) & $a=-1,477$ & 0,6003 \\
& $b=3004$ & \\
Quadrático (2 ${ }^{\mathrm{a}}$ ordem) & $a=-0,1818529042$ & 0,8410 \\
& $b=728,2492729425$ \\
& $c=-729022,310424804$ \\
\hline
\end{tabular}

O coeficiente $a$ é a taxa de variação anual da linha de costa para cada setor, ou seja, é a velocidade em metros por ano (m/ano) que a linha de costa varia, onde coeficientes negativos indicam erosão e positivos acresção.

A aplicação do MMQ para o cálculo dos parâmetros conduziu a valores de $\mathrm{R}^{2}$ mais próximos de um (1) para o modelo polinomial quadrático. Ou seja, para este exemplo indica-se que o modelo quadrático se ajusta melhor ao conjunto de observações, porém não significa dizer que por este motivo ele pode ser considerado como o melhor modelo para verificar a tendência das observações, como será indicado no experimento a seguir.

Os valores da tendência obtidos através dos modelos polinomiais ajustados às amostras do setor norte e sul, como também o valor em módulo das discrepâncias, obtidas na comparação entre o "valor verdadeiro" de 2015 e os valores de tendência, são mostrados nas Tab. 5 (norte) e Tab. 6 (sul).

Tabela 5 - Valores da tendência obtidos através dos modelos polinomiais ajustados e o módulo das discrepâncias entre o "dado real" de 2015 e estes valores de tendência para o setor norte.

\begin{tabular}{ccc}
\hline \multicolumn{3}{c}{ SETOR NORTE } \\
\hline Modelo & Tendência 2015 $(\mathrm{m})$ & Discrepância $(\mathrm{m})$ \\
\hline Linear $\left(1^{\mathrm{a}}\right.$ ordem $)$ & 27,2 & 3,6 \\
Quadrático $\left(2^{\mathrm{a}}\right.$ ordem $)$ & 32,5 & 8,9 \\
\hline
\end{tabular}

Tabela 6 - Valores da tendência obtidos através dos modelos polinomiais ajustados e o módulo das discrepâncias entre o "valor verdadeiro" de 2015 e estes valores de tendência para o setor sul.

\begin{tabular}{|c|c|c|}
\hline \multicolumn{3}{|c|}{ SETOR SUL } \\
\hline Modelo & Tendência $2015(\mathrm{~m})$ & Discrepância (m) \\
\hline Linear $\left(1^{\mathrm{a}}\right.$ ordem $)$ & 27,8 & 3,0 \\
\hline Quadrático $\left(2^{\mathrm{a}}\right.$ ordem $)$ & 36,2 & 11,4 \\
\hline
\end{tabular}

Observa-se que as tendências calculadas pelo modelo linear foram menos disparas que as alcançadas pelo modelo quadrático, quando comparadas ao valor considerado como "dado real" do ano 2015.

Os valores de tendência para os anos de 2020, 2025, 2030, 2035, 2040, para os dois setores, norte e sul, e são mostradas na Fig. 5 e Fig. 6, respectivamente.

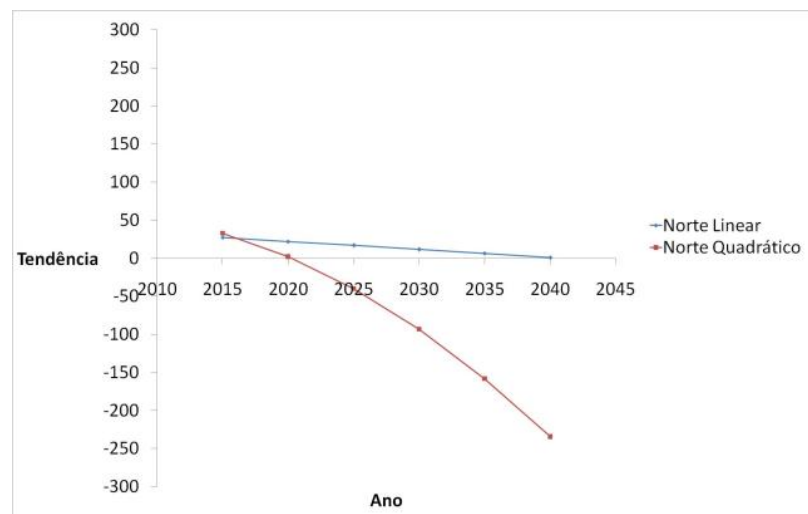

Figura 5 - Setor norte: Valores de tendência da linha de costa em metros VS ano.

Fonte: $\mathrm{O}$ autor.

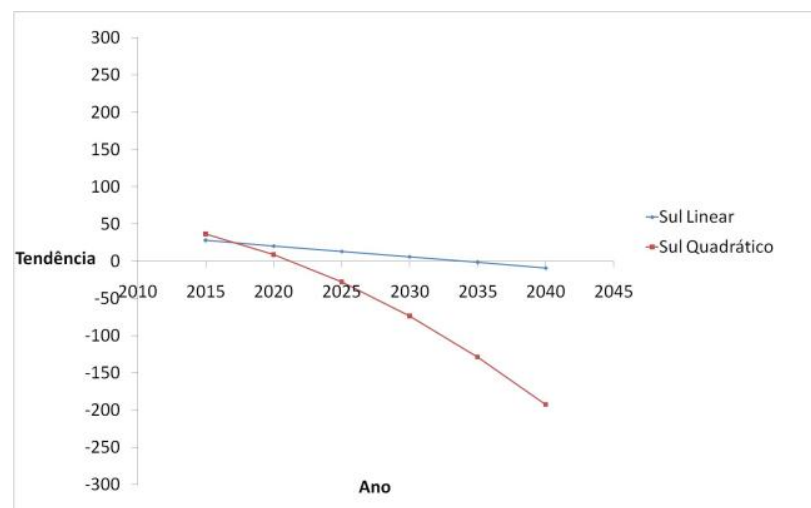

Figura 6 - Setor sul: Valores de tendência da linha de costa em metros VS ano.

Fonte: $\mathrm{O}$ autor.

Nas fig. 5 e 6 é possível verificar que a escolha de um modelo de tendência para representar o comportamento futuro da linha de costa é fundamental e não deve ser baseada apenas em um modelo matemático que se ajusta da melhor forma possível a um conjunto de observações. Observa-se nas figuras que a medida que o tempo aumenta a tendência de resposta posicional da linha de costa vai se afastando chegando a aproximadamente 200 metros de diferença entre os modelos independente do setor analisado.

$\mathrm{Na}$ literatura encontram-se previsões consideradas de curto, médio e longo prazo (DEMAREST; LEATHERMAN, 1985; GALGANO et al., 1998; GALGANO; DOUGLAS, 2000; FENSTER et al., 2000). A previsão de curto prazo pode ser de alguns meses ou anos, a de médio prazo em torno de 10 a 70 anos e a de longo prazo de 100 a 1000 anos ou mais dependendo do estudo proposto, principalmente utilizado para períodos geológicos. Por exemplo, em Appeaning Addo et al (2008), foi efetuado uma previsão considerando um 
horizonte de 250 anos. Neste estudo proposto o mais importante foi destacar que diferentes modelos geram diferentes tendências, e por isso é necessário um cuidado especial na escolha de um modelo matemático e probabilístico para representar um fenômeno. Destaca-se a importância do monitoramento e alimentação de informações de entrada para que o modelo proposto possa ser atualizado na medida do possível e desta forma represente o fenômeno estudado.

\section{CONCLUSÕES}

Como pode ser visto nos dados apresentados neste trabalho, a linha de costa é dinâmica, possuindo variações consideráveis, na ordem de metros por ano, exigindo que os gestores estejam munidos de informações acerca do seu comportamento, para evitar que, por exemplo, sejam construídos aparatos urbanos de lazer ou moradia a uma distância inapropriada da área de influência da LC.

Observa-se que as discrepâncias entre as predições calculadas pelo modelo linear foram menores que as alcançadas pelo modelo quadrático, nos dois setores.

Por outro lado, os valores de $\mathrm{R}^{2}$ calculados para os dois modelos foram melhores para $\mathrm{o}$ polinomial quadrático, pois estão mais próximos a 1 , o que aponta que os resíduos obtidos no ajuste da função são menores para este modelo, e a função foi melhor ajustada ao modelo quadrático do que ao modelo linear.

Porém, deve-se considerar a não-uniformidade nas variações das distâncias ao longo do trecho sul, que apresentou uma discrepância maior. Portanto, é necessária cautela ao tentar predizer a posição da linha de costa para uma determinada área de estudo. Não basta apenas a escolha de um modelo matemático (regressão linear ou quadrática das observações), é necessário considerar outros fatores como a quantidade de amostras utilizadas no modelo, frequência de ocorrências de tempestades e impactos desses acontecimentos na área estudada.

Por fim salienta-se a importância em investimentos e apoio a pesquisas que busquem aplicar técnicas de monitoramento costeiro sejam elas oriundas do sensoriamento remoto tais como: imagens de satélites, aerofotogrametria, Light Detection And Ranging (LIDAR), videometria ou GNSS.

\section{AGRADECIMENTOS}

Nesta seção os autores expressam seus agradecimentos aos membros do projeto de pesquisa de Monitoramento Geodésico da Linha de Costa da Praia do Seixas, do Laboratório de Geoprocessamento do Instituto Federal da Paraíba, Campus João Pessoa, pela cessão dos dados utilizados neste trabalho. À Coordenação de Aperfeiçoamento de Pessoal de Nível Superior (CAPES) pela bolsa de mestrado ao primeiro autor, bem como ao Programa de Pós-Graduação em Ciências Geodésicas e Tecnologias da Geoinformação, da Universidade Federal de Pernambuco. Também aos revisores da Revista Brasileira de Geomática, pelos importantes comentários e sugestões.

\section{REFERÊNCIAS BIBLIOGRÁFICAS}

APPEANING ADDO, K.; WALKDEN, M.; MILLS, J. P. Detection, measurement and prediction of shoreline recession in Accra, Ghana. ISPRS Journal of Photogrammetry and Remote Sensing, v. 63, n. 5, p. 543-558, 2008.

BOAK, E. H.; TURNER, I. L. Shoreline Definition and Detection: A Review. Journal of Coastal Research. 21(4), P. 688-703, 2005.

CROWELL, M.; LEATHERMAN, S.P.; BUCKLEY, M.K. Shoreline Change Rate Analysis: Long Term versus Short Term Data. Shore and Beach. v.61, n.1, 13-20, 1993.

CURRIN, C.; DAVIS, J.; COWART BARON, L.; MALHOTRA, A.; FONSECA, M. Shoreline change in the New River Estuary, North Carolina: Rates and consequences. Journal of Coastal Research, 31(5), 2015. 1069-1077.

DAN, H; YONGJIU, F; LIJUN, S. Mapping Shoreline of Hangzhou Bay with Remote Sensing Images from 1979 to 2005. Remote Sensing, 2012. Disponível em: $<$ http://ieeexplore.ieee.org/xpls/abs_all.jsp?arnumber $=6260621>$.

DEMAREST, J. M.; LEATHERMAN, S. P. Mainland influence on coastal transgression: Delmarva Peninsula. Marine Geology, v. 63, n. 1, p. 19-33, 1985.

DOLAN, R.B.; HAYDEN, B.; MAY, P.; MAY, S. The reliability of shoreline change measured from aerial photographs. Shore \& Beach, 48, 1980. 22-29.

DOUGLAS, B. C.; CROWELL, M. Long-term Shoreline Position Prediction and Error Propagation. Journal of Coastal Research, v. 16, n. 1, 2012.

FENSTER, M. S.; DOLAN, R.; MORTON, R. A. Coastal storms and shoreline change: signal or noise? Journal of Coastal Research, p. 714-720, 2001.

GALGANO, Francis A.; DOUGLAS, Bruce C.; LEATHERMAN, Stephen P. Trends and variability of shoreline position. Journal of Coastal Research, p. 282-291, 1998.

GALGANO, F. A.; DOUGLAS, B. C. Shoreline position prediction: methods and errors. Environmental Geosciences, v. 7, n. 1, p. 23-31, 2000.

GEMAEL, Camil. Introdução ao ajustamento de observações: aplicações geodésicas. Editora UFPr, 1994.

GENZ, A. S.; FLETCHER, C. H.; DUNN, R. A.; FRAZER, L. N.; ROONEY, J. J. The Predictive Accuracy of Shoreline Change Rate Methods and Alongshore Beach Variation on Maui, Hawaii. Journal of Coastal Research, v. 231, 2007, p. 87105.

GONÇALVES, R. M. Modelagem de tendência a curtoprazo da linha de costa através de dados geodésicos temporais utilizando regressão linear, estimativa robusta e redes neurais artificiais. Tese de conclusão de curso (Doutorado em Ciências Geodésicas), UFPR, 2010. 152p. 
GONÇALVES, R. M.; AWANGE, J.; KRUEGER, C. P.; HECK, B.; COELHO, L. S. A comparison between three short-term shoreline prediction models. Ocean \& Coastal Management, v. 69, 2012, p. 102-110.

GONÇALVES, R. M.; COELHO, L. D. S; KRUEGER, C. P.; HECK, B. Modelagem preditiva de linha de costa utilizando redes neurais artificiais. Boletim de Ciências Geodésicas (Impresso), v.16, 2010, p.420 444.

LEATHERMAN, S. P.; CLOW, B. UMD shoreline mapping project. IEEE Geoscience and Remote Sensing Society Newsletter, v. 22, n. 3, p. 5-8, 1983.

LI, R.; MA, R.; DI, K. Digital Tide-Coordinated Shoreline. Marine Geodesy, v. 25, n. 1-2, p. 27-36, 2002.

MANCA, E., PASCUCCI, V., DELUCA, M., COSSU, A., ANDREUCCI, S. Shoreline evolution related to coastal development of a managed beach in Alghero, Sardinia, Italy. Ocean \& Coastal Management 85, 2013, 65-76.

MENDONCA, F. J. B., GONÇALVES, R. M., AWANGE, J., SILVA, L. M., GREGORIO, M. N. TEMPORAL SHORELINE SERIES ANALYSIS USING GNSS. Boletim de Ciências Geodésicas (Online), v.20, 2014, p.701 - 719.

REIS, M. C. O litoral de João Pessoa (PB), frente ao problema da erosão costeira. Tese de conclusão de curso (Doutorado em Geociências), UFPE, 2008. $131 \mathrm{p}$.

UMAR, Z., AKIB, W.A.A.W.M., AHMAD, A. Monitoring shoreline change using Remote sensing and GIS: A case study of padang coastal area, Indonesia. IEEE 9th International Colloquium on Signal Processing and its Applications. 2013.

WHITE, S., 2007. Utilization of LIDAR and NOAA's Vertical Datum Transformation Tool (VDatum) for Shoreline Delineation. Oceans. 2007. 